\title{
Fosfomycin to Control Contamination in M. tuberculosis Culture in BACTEC MGIT 960 System
}

\author{
Roger Ivan Calderon Espinoza ${ }^{1,2}$, Maria Belen Arriaga Gutierrez ${ }^{3,4,5}$, Kattya Lopez Tamara ${ }^{1}$, \\ Nadia Nilda Barreda Ponce ${ }^{1}$, Carole Diane Mitnick ${ }^{6}$, Geraint Rhys Davies ${ }^{7}$, David John Coleman ${ }^{1}$ \\ ${ }^{1}$ Socios En Salud Sucursal Peru, Lima, Peru \\ ${ }^{2}$ Faculdade de Medicina, Universidade Federal do Rio de Janeiro, Rio de Janeiro, Brazil \\ ${ }^{3}$ Faculdade de Medicina, Universidade Federal da Bahia, Bahia, Brazil \\ ${ }^{4}$ Instituto Gonçalo Moniz, Fundação Gonçalo Cruz, Bahia, Brazil \\ ${ }^{5}$ Instituto Brasileiro para Investigação da Tuberculose (IBIT), Bahia, Brazil \\ ${ }^{6}$ Department of Global Health and Social Medicine, Harvard Medical School, Boston, USA \\ ${ }^{7}$ Institutes of Infection and Global Health \& Translational Medicine. University of Liverpool, Liverpool, United Kingdom
}

Email address:

rcalderon_ses@pih.org (R. I. C. Espinoza)

\section{To cite this article:}

Roger Ivan Calderon Espinoza, Maria Belen Arriaga Gutierrez, Kattya Lopez Tamara, Nadia Nilda Barreda Ponce, Carole Diane Mitnick, Geraint Rhys Davies, David John Coleman. Fosfomycin to Control Contamination in M. tuberculosis Culture in BACTEC MGIT 960 System. European Journal of Clinical and Biomedical Sciences. Vol. 4, No. 3, 2018, pp. 51-54. doi: 10.11648/j.ejcbs.20180403.13

Received: July 9, 2018; Accepted: August 12, 2018; Published: September 12, 2018

\begin{abstract}
The BACTEC MGIT 960 system is widely used for recovery of Mycobacterium tuberculosis, but high contamination rates compromise its diagnostic performance. Efforts to control contamination must balance elimination of contaminating organisms while preserving growth of tuberculous bacilli. Fosfomycin has activity against many bacteria responsible for culture contamination yet is inactive against Mycobacterium tuberculosis. Therefore, it holds potential as a selection agent for the culture of $M$. tuberculosis clinical samples with the MGIT system. In this study, we assess the ability of fosfomycin supplement to MGIT cultures of clinical sputum specimens. Sputum specimens collected at 62 diagnosis and treatment monitoring visits during a randomized controlled trial of high-dose rifampin (ClinicalTrials.gov NCT01408914) were split: one-half was treated as conventional and the other half was treated with $1 \mathrm{mg}$ of fosfomycin. The frequencies of contamination and of recovery of $M$. tuberculosis were compared using McNemar and Wilcoxon signed-rank test, respectively, with $\alpha=0.05$. In the fosfomycin-treated samples, $2 / 62$ (3.2\%) yielded contaminated cultures while $12 / 62(19.4 \%)$ of untreated cultures were contaminated $(\mathrm{p}<0.001$, McNemar Test). Detection of $M$. tuberculosis was not significantly altered by the addition of fosfomycin (Time to positivity, $\mathrm{p}=0.576$, Student's $\mathrm{T}$ Test; log colony forming units), nor the Colony Forming Unit Counts (Wilcoxon signed-rank test $\mathrm{p}=0.671$ ). These results suggest that the use of fosfomycin to control contamination does not affect the natural growth of MTB. Its use could result in a significant improvement in recovery of M. tuberculosis in MGIT.
\end{abstract}

Keywords: Fosfomycin, Culture Contamination, Tuberculosis, BACTEC MGIT ${ }^{\mathrm{TM}} 960$

\section{Introduction}

The BACTEC Mycobacteria Growth Indicator Tube ${ }^{\mathrm{TM}} 960$ System (MGIT) is an advanced diagnostic tool commonly employed in laboratories worldwide for mycobacteria recovery from clinical specimens, most notably for Mycobacterium tuberculosis (MTB). However, sometimes very high contamination rates have been observed MGIT cultures from sputum samples from TB patients,[1] reducing its promise as an effective diagnostic tool.

MGIT uses antibiotic mix knowns as PANTA - polymyxin $\mathrm{B}$, amphotericin $\mathrm{B}$, nalidixic acid, trimethoprim and azlocillin [2], Contamination persists, however, in PANTA-treated specimens [3]. Some strategies to decrease the contamination rate of cultures have been have explored: through specific refrigeration methods [4], sputum pre-treatment [5], bacteriophage targeted approaches [6], sodium hydroxide/ 
effective at $\mathrm{N}$-acetyl-cysteine treatment [7], and cetylpyridinium bromide or cetylpyridinium chloride preservations $[3,8]$. The use of selective agents as oral rinses prior to sputum collection also has been tested [9]. Of these, none has proven reliable, inexpensive, easily integrated into sample collection or processing, and significantly reducing culture contamination. Fosfomycin is a broad spectrum bactericidal antibiotic $[10,11]$ of the phosphonic group. It was first discovered in Streptomycin species cultures supernatants in Spain in 1969 [12]. It inhibits the cytoplasmic enzyme phosphoenolpyruvate transferase that catalyzes the formation of uridine diphosphate- $\mathrm{N}$-acetylmuramic acid during the first step of peptidoglycan synthesis [13]. Access into the cell requires the use 1- $\alpha$-glycero-phosphate transport system or the hexose-monophosphate transport system. The Actinobacteria, which include genera Mycobacterium and Micrococcus, do not have these transport systems and are natural resistant because of the lack of penetration. [14]

For these reasons, we assessed whether fosfomycin added to MTB cultures could prevent culture contamination in MGIT.

\section{Methods}

\subsection{Patients}

Study samples were drawn from all sputum specimens smear-positive for MTB among patients being screened or treated for tuberculosis at two public hospitals in Lima, Peru, between February and April of 2015.

\subsection{Sample Size Calculations}

Sample size was calculated (OpenEpi ver. 3.01) to detect the difference between contamination in $15.4 \%$ of fosfomycin-untreated cultures (as observed in our lab) and less than $5 \%$ contamination in fosfomycin-treated cultures, with $80 \%$ power and $95 \%$ confidence. The estimated sample size was 62-paired (fosfomycin-treated and -untreated) samples.

\subsection{Specimen Processing}

Specimens were transported on the day they were collected to the study laboratory, the BSL-3 laboratory of the Peruvian non-governmental organization, Socios En Salud. They were processed for smear and culture in MGIT using procedures described for the manufacturer [2] using PANTA. In brief, the sputum specimens were treated with NALC-NaOH at $2 \%$ and neutralized with phosphate-buffered saline $\mathrm{pH} 6.8$ 0.067M. Each sample was divided into two aliquots for inoculation into MGIT i) as reference culture and ii) culture with $1 \mathrm{mg}$ of fosfomycin disodium salt. (Sigma-Aldrich P5396 CAS \# 20016-99-9). The time to positivity (TTP) for MTB was recorded for all cultures, and upon a positive culture result, the colony forming unit (CFU) counts were also recorded from the MGIT system. To confirm if the observed growth was MTB or contaminating non-tuberculous bacteria, $10 \mathrm{uL}$ of cultured media growth from the MGIT culture were inoculated on 5\% sheep blood agar plates. Additionally, one smear from each MGIT culture was analyzed to identify the presence of acid-fast bacilli (AFB). Any growth of bacteria exhibited on blood agar plates was resulted in classification of culture as contaminated. In the presence of AFB and the absence of growth on blood agar plates, the culture was considered positive. After confirmation of AFB presence, the identification of the MTB complex was performed using BD MGIT ${ }^{\mathrm{TM}} \mathrm{TBc}$ Identification Test (BD 245159) using $100 \mu \mathrm{L}$ of positive cultures. Cultures without signal of mycobacterial growth after 42 days were classified as negative for MTB.

\subsection{Data Analysis}

Comparison of results of contamination and culture positivity between paired fosfomycin-treated and fosfomycin-untreated cultures were performed by McNemar test. Difference in time to TTP was evaluated using the paired sample Student's T-test; the Wilcoxon signed-rank test was used to assess the difference in the CFU counts between the both groups. Those tests yielding $\mathrm{p}<0.05$ were considered statistically significant.

\section{Results}

During the study period, 62 sputum specimens were collected. Sample volume and appearance are described in Table 1.

Table 1. Description of appearance and volume of study samples (by result in untreated culture) processed at the laboratory of Socios En Salud Sucursal Peru, Lima Peru between February and April of 2015.

\begin{tabular}{|c|c|c|c|c|}
\hline Result & $\begin{array}{l}\text { Positive culture } \\
\mathrm{N}=35\end{array}$ & $\begin{array}{l}\text { Contaminated Culture } \\
\mathrm{N}=15\end{array}$ & $\begin{array}{l}\text { Negative Culture } \\
\mathrm{N}=12\end{array}$ & $\begin{array}{l}\text { Total cultures } \\
\mathrm{N}=62\end{array}$ \\
\hline Salival* N (\%) & $14(40.0)$ & $4(33.3)$ & $8(53.3)$ & $26(41.9)$ \\
\hline Mucous, purulent or mucopurulent* N (\%) & $21(60.0)$ & $8(66.7)$ & $7(46.7)$ & $36(58.1)$ \\
\hline $\begin{array}{l}\text { Sample volume }(\mathrm{mL}) * * \\
\text { Median }(\mathrm{IQR}) * * *\end{array}$ & $8.0(19.0)$ & $10.0(34.0)$ & $7.0(31.0)$ & $7.5(15.0)$ \\
\hline
\end{tabular}

$* \mathrm{p}=0.54(\chi 2) . * * \mathrm{p}=0.83$ (Kruskal-Wallis tests). $* * *$ IQR: Interquartile range

Among these, 56.5\% (35/62) of samples were positive for MTB in MGIT culture not treated with fosfomycin; $40 \%$ $(14 / 35)$ of them had saliva appearance. The median sample volume was $7.5 \mathrm{~mL}$. On visual inspection, there was no significant difference in volume or characteristics across culture results.

Table 2 shows the comparison of results between the fosfomycin-untreated and -treated cultures. Only two (3.2\%) 
cultures treated with fosfomycin were contaminated while $12 \quad(19.4 \%)$ untreated cultures were contaminated ( $\mathrm{p}<0.001)$.

Table 2. Results of BACTEC MGIT 960 cultures: untreated and treated with fosfomycin.

\begin{tabular}{llll}
\hline Result & $\begin{array}{l}\text { Culture not treated with } \\
\text { fosfomycin (N=62) }\end{array}$ & $\begin{array}{l}\text { Culture treated with } \\
\text { fosfomycin (N=62) }\end{array}$ & $\boldsymbol{p}$ value* \\
\hline Culture positive for MTB- $\mathrm{n}(\%)$ & $35(56.5)$ & $45(72.6)$ & $0.002^{*}$ \\
Culture contaminated with nontuberculous bacteria $-\mathrm{n}(\%)$ & $12(19.4)$ & $2(3.2)$ & $0.001^{*}$ \\
TTP (hours) Mean \pm Standard Deviation & $363.9 \pm 193.6$ & $359.9 \pm 173.4$ & $0.576^{* *}$ \\
CFU counts (per mL) Median (IQR) & $151(269)$ & $169(421)$ & $0.456^{* * *}$ \\
\hline
\end{tabular}

*McNemar Test, **Student's t Test for related samples, ${ }^{* * * W i l c o x o n ~ s i g n e d-r a n k ~ T e s t ~}$

Among the 12 contaminated, initially fosfomycinuntreated cultures, $M$. tuberculosis recovery was achieved in $10(83.3 \%)$ after treatment with fosfomycin. TPP and CFU counts did not differ between the conventional treatment and fosfomycin treatment groups.

\section{Discussion}

The addition of fosfomycin could reduce the contamination in more than six times in MGIT cultures; without affect the grow characteristics as TTP and CFU levels. As we know, the MGIT BACTEC 960 system enhances recovery of mycobacteria in various types of clinical specimens compared with culture in solid media, such as LJ $[15,16]$ and other liquid media [17]. However, according to our results, the variable contamination rates in sputum samples from patients diagnosed with tuberculosis, we effective controlled as only the addition of fosfomycin.

Following the sense of improving diagnostic tools as reported by other groups, as we have reported a large rate of contamination in cultures (around 20\%), we decided to implement a low cost measure that allows us to reduce the contamination without compromising the isolation ability of MGIT System (CFU counts obtained during incubation) or time to positivity cultures, being major quality variables. It should be noted that several studies have been published referring to variable rates of contamination, as in the case of Whyte et al,[1] were able to achieve up to $30 \%$ of the analyzed samples. In that sense, the original decontamination protocol has not been modified substantially and the volume of analyzed sputum samples was never greater than $3 \mathrm{~mL}$.

Since the comparative study described here performed on all samples received for culture MGIT 960, a variable to consider is the appearance of sputum, despite its expected characteristics (mucus and pus) that could hinder the rapid diagnosis the tuberculosis [18], contamination could be caused by the presence of saliva, which would be the main source of gram-positive flora that escapes the action of chemical decontamination and its growth could masks the $M$. tuberculosis growth [19]. Table N 1 indicates that the volume and the appearance of the sputum specimen had not significant differences to obtain positive, negative or contaminated results in the culture.

Despite what some reports indicate, PANTA suppresses the growth of contaminating bacteria in various liquid culture methods, such as MGIT 960, and may have an inhibitory effect on the growth of some mycobacteria [2], coinciding with what was found in our experiments and even with the addition of fosfomycin. The average of time to positivity is inversely proportional to the level of smear positivity of samples; but could be up to 16 days [20]. In the samples analyzed, the average of TTP were about 15 days, reflecting a low level of positivity in the samples, so the risk of contamination due to the oral flora of patients could be higher. However, the addition of fosfomycin, did not affect the value of TTP or CFU expected (in agree with the control). Contamination can result from a number of causes, such as the host co-infections, clinical heterogeneity of populations from which the samples are collected, or delayed the transport of samples to the laboratory, allowing microbial proliferation. Clinical sputum samples are contaminated with oral microflora, but the bacterial counts are usually less than $10^{7}$ organisms $/ \mathrm{ml}$ [21], with higher counts associated with contamination failures.

According to the list price of fosfomycin as sodium salt, the addition of $1 \mathrm{mg}$ per MGIT tube cost about USD 0.02 per sample, which in our opinion is a negligible change in the price of performing MGIT culture.

On other side, this in vitro procedure does not confront nontuberculous organisms in vivo with fosfomycin; so we will not generate a selective pressure that generates resistance to this drug. Also, since MTB is innate resistant to this drug, it has been shown that the concentration of fosfomycin is a safer, and higher form of contamination control management other organisms or substances. The strategy of using oral rinse with chlorhexidine gluconate and cetylpyridinium chloride [9] or specimen preservations [8] to reduce contamination showed lower results than ours. Still, we consider the remaining exposure of oral organisms can become a future risk for patients and usefulness of this method. In other sides, like using phages [6], the potential risk in laboratories to work in other microorganisms is high, with full exclusive use in expert laboratories MTB with exclusive BSL3 conditions.

\section{Conclusions}

The strategy of adding fosfomycin into MGIT 960 cultures require a proper validation. However, our findings allow us to consider that it could be a simple and inexpensive method to control the contamination of cultures without affect bacterial growth patterns or interfering the culture grow. This strategy is inexpensive and could be available in many developing countries, where the performance of diagnostic confirmation system - culture based do not should affected 
by the contamination problems. Therefore, this will improve the usefulness of the TB culture in treatment decisions us to continue with the following tests of the diagnostic algorithm (such as drug susceptibility tests) and thus control the health of affected people and steadily improve the quality of life of the population of the region.

\section{Acknowledgements}

We thank the health care workers at Hospital Nacional Sergio Bernales and Hospital Nacional Hipolito Unanue. We especially thank the patients and families who made this study possible.

\section{Supported by}

NIH/NIAID grant U01 AI091429 to C.D.M. and G.R.D.

\section{References}

[1] Whyte T, Cormican M, Hanahoe B, Doran G, Collins T, Corbett-Feeney G. Comparison of BACTEC MGIT 960 and BACTEC 460 for culture of Mycobacteria. Diagn Microbiol Infect Dis. 2000;38(2):123-126.

[2] Siddiqi SH, Rüsch-Gerdes S. MGIT $T^{T M}$ Procedure Manual. Geneva, Switzerland; 2006.

[3] Lumb R, Ardian M, Waramori G, et al. An alternative method for sputum storage and transport for Mycobacterium tuberculosis drug resistance surveys. Int J Tuberc Lung Dis. 2006;10(2):172-177.

[4] Banda HT, Harries A, Boeree MJ, Nyirenda TE, Banerjee A, Salaniponi FML. Viability of stored sputum specimens for smear microscopy and culture. Int $J$ Tuberc Lung Dis. 2000;4(3):272-274.

[5] Azam K, Cadir N, Madeira C, Gillespie SH, Sabiiti W. OMNIgene. SPUTUM suppresses contaminants while maintaining Mycobacterium tuberculosis viability and obviates cold-chain transport. ERJ Open Res. 2018;4(1):00074-2017. doi:10.1183/23120541.00074-2017.

[6] Kumar V, Balaji S, Gomathi NS, et al. Phage cocktail to control the exponential growth of normal flora in processed sputum specimens grown overnight in liquid medium for rapid TB diagnosis. J Microbiol Methods. 2007;68(3):536-542. doi:10.1016/j.mimet.2006.10.015.

[7] Kelly PM, Lumb R, Pinto A, da Costa G, Sarmento J, Bastian I. Analysis of Mycobacterium tuberculosis isolates from treatment failure patients living in East Timor. Int $J$ Tuberc Lung Dis. 2005;9(1):81-86. doi:10.1016/j.eimc.2018.05.009.

[8] Ardizzoni E, Mulders W, Sanchez-Padilla E, Varaine F, De Jong BC, Rigouts L. Decontamination methods for samples preserved in cetylpyridinium chloride and cultured on thinlayer agar. Int J Tuberc Lung Dis. 2014;18(8):972-977. doi:10.5588/ijtld.13.0887.
[9] Peres RL, Palaci M, Loureiro RB, et al. Evaluation of oral antiseptic rinsing before sputum collection to reduce contamination of mycobacterial cultures. $J$ Clin Microbiol. 2011;49(8):3058-3060. doi:10.1128/JCM.00541-11.

[10] Lifonzo-Mucha S, Tamariz-Zamudio P, Champi-Merino R. Sensibilidad a Fosfomicina en Escherichia coli productoras de Betalactamasas de Espectro extendido. Rev Peru Med Exp Salud Publica. 2015;35(1):68-71.

[11] Coronado-Álvarez NM, Parra D, Parra-Ruiz J. Clinical efficacy of fosfomycin combinations against a variety of gram-positive cocci. Enferm Infecc Microbiol Clin. 2018. doi:10.1016/j.eimc.2018.05.009.

[12] Raz R. Fosfomycin: An old-new antibiotic. Clin Microbiol Infect. 2012;18(1):4-7.

[13] Kahan FM, Kahan JS, Cassidy PJ. The mechanism of action of Fosfomycin (Phosphonomycin). Ann NY Acad Sci. 1974;235:364-386.

[14] Brown AC, Parish T. Dxr is essential in Mycobacterium tuberculosis and fosmidomycin resistance is due to a lack of uptake. BMC Microbiol. 2008;8(1):78. doi:10.1186/14712180-8-78.

[15] Lu D, Heeren B, Dunne WM. Comparison of the automated Mycobacteria Growth Indicator Tube system (BACTEC 960/MGIT) with Lowenstein-Jensen medium for recovery of mycobacteria from clinical specimens. Am J Clin Pathol. 2002;118(4):542-545.

[16] Yan JJ, Huang AH, Tsai SH, Ko WC, Jin YT, Wu JJ. Comparison of the MB/BacT and BACTEC MGIT 960 system for recovery of mycobacteria from clinical specimens. Diagn Microbiol Infect Dis. 2000;37(1):25-30. doi:10.1016/S07328893(00)00118-8.

[17] Feyzioglu B, Dogan M, Sanli OO, Ozdemir M, Baykan M. Comparison of the performance of TK system with LJ and MGIT methods in the diagnosis of tuberculosis. Int J Clin Exp Med. 2014;7(4):1084-1088.

[18] Park DJ, Drobniewski F, Meyer A, Wilson SM. Use of a phage-based asay for penotypic detection of mycobacteria directly from sputum. J Clin Microbiol. 2003;41(2):680-688.

[19] Cornfield DB, Beavis KG, Greene JA, Bojak M, Bondi J. Mycobacterial growth and bacterial contamination in the mycobacteria growth indicator tube and BACTEC 460 culture systems. J Clin Microbiol. 1997;35(8):2068-2071.

[20] Rodrigues C, Shenai S, Sadani M, et al. Evaluation of the BACTEC MGIT 960 TB system for recovery and identification of Mycobacterium tuberculosis complex in a high through put tertiary care centre. Indian J Med Microbiol. 2009;27(3):217-221. doi:10.4103/0255-0857.53203.

[21] Tebbutt GM, Coleman DJ. Evaluation of some methods for the laboratory examination of sputum. J Clin Pathol. 1978;31(December 1977):724-729.

[22] Kumar V, Balaji S, Gomathi NS, et al. Phage cocktail to control the exponential growth of normal flora in processed sputum specimens grown overnight in liquid medium for rapid TB diagnosis. J Microbiol Methods. 2007;68(3):536-542. 